Revue d'histoire de l'Amérique française

सES REVUE D.HISTOIRE DE L'AMÉRIQUE FRANÇAISE

\title{
La difficile érection du diocèse de Montréal (1836)
}

\section{Léon Pouliot}

Volume 16, numéro 4, mars 1963

URI : https://id.erudit.org/iderudit/302228ar

DOI : https://doi.org/10.7202/302228ar

Aller au sommaire du numéro

Éditeur(s)

Institut d'histoire de l'Amérique française

ISSN

0035-2357 (imprimé)

1492-1383 (numérique)

Découvrir la revue

Citer cet article

Pouliot, L. (1963). La difficile érection du diocèse de Montréal (1836). Revue

d'histoire de l'Amérique française, 16(4), 506-535.

https://doi.org/10.7202/302228ar d'utilisation que vous pouvez consulter en ligne.

https://apropos.erudit.org/fr/usagers/politique-dutilisation/ 


\section{LA DIFFICILE ÉRECTION DU DIOCĖSE DE MONTRÉAL (1836)}

\section{LE PROBLEME}

Dans Monseigneur Bourget et son Temps, nous avons dit la condition singulière de $\mathrm{Mgr}$ Jean-Jacques Lartigue, évêque à Montréal, mais non évêque de Montréal, de 1821 à 1836. Vicaire général, revêtu du caractère épiscopal et suffragant de Québec, il est, dans la pensée de Rome, le dédoublement parfait à Montréal de l'évêque de Québec ${ }^{1}$. Mais cette qualité lui est contestée par une partie du clergé et des fidèles; de plus, elle est délibérément ignorée de l'autorité civile. Il résulte de là que l'évêque de Telmesse est impuissant devant les cabales et les intérêts qui se liguent contre lui. Ajoutons à cela que, malgré une rectitude d'intention et une sainteté peu communes, il est d'un commerce difficile, surtout quand il soutient les droits ou ce qu'il croit être les droits imprescriptibles de l'Église.

Aussi, cet homme d'une force de volonté incomparable a, plus d'une fois, cédé au découragement. Il écrivait au Préfet de la Propagande, le 19 octobre 1832:

Tout cela me dégoûte du ministère infructueux qui m'a été imposé par le Saint-Siège ${ }^{2}$; parce que je vois clairement depuis plus de douze ans que je l'exerce, qu'un Evêque ne peut faire presque aucun bien, surtout à cause de l'opposition systématique des Sulpiciens étrangers, qui malheureusement sont protégés à Rome par des hommes puissants, et que je crois bien intentionnés, mais qui ne connaissent aucunement le véritable état de nos affaires ecclésiastiques; c'est pourquoi j'insiste de nouveau, et plus que jamais, sur l'acceptation par le Tr. St-Père de ma démission comme Suffragant pour Montréal ${ }^{3}$.

${ }^{1} \mathrm{Mgr}$ Bourget et son Temps, I, chapitre préliminaire: 21-40.

2 Mgr Lartigue n'avait accepté l'épiscopat que sur un ordre in virtute sanctro obodientiix, venu de Rome.

3 Prop. Acta, 1833. 
A sa réunion du 25 février 1833, la Propagande décidait d'exaucer le vœu de Mgr Lartigue et de le remplacer à Montréal par un vicaire général non revêtu du caractère épiscopal ${ }^{4}$. Décision malheureuse ! C'était le retour à un état de choses que, dès $1820, \mathrm{Mgr}$ Plessis jugeait inadapté à la réalité, inefficace et préjudiciable au bien des âmes. Et l'on pense bien qu'en ces douze dernières années, le besoin d'un évêque à Montréal n'a fait que croître. Qui viendra au secours de Montréal ? Ancien préfet de la Propagande, Grégoire XVI était au courant du problème. En 1829, il avait entendu les plaintes soumises, mais combien douloureuses des députés à Rome de Mgr Lartigue, MM. Thomas Maguire et Antoine Tabeau. En 1832, il avait écrit de sa main à l'évêque de Telmesse. ${ }^{5}$ Enfin, cette réunion du 25 février s'étant tenue en sa présence ${ }^{6}$, il en connut, dès la première heure, toutes les décisions et il en mesura toute la gravité. Aussi, quand le secrétaire de la Propagande vint les présenter à son approbation, il refusa de les signer ${ }^{7}$. Une lettre adressée à Mgr Lartigue rappelait la réunion du 25 février; mais elle en taisait les décisions et elle y substituait la volonté du Pape: «Toutes choses bien considérées, Sa Sainteté a ordonné de vous signifier, en son nom, qu'elle désire vous voir persévérer dans votre poste ${ }^{8}$.»

\section{Ibid.}

5 Prop. Scrit. rif., 1833, 113-114. Mgr Lartigue au cardinal préfet, 19 octobre 1832. Il lui demande d'obtenir deux choses du Pape, «qui a eu, l'été dernier, la condescendance de m'honorer d'une lettre écrite de sa main 》.

6 Prop. Acta, 1834, 63. « Nel palazzo apostolico vaticano al cospetto di Nostro Signore Gregorio PP. XVI, si tenne la congregazione generale di Propaganda, il giorno 25 Febbraio $1833 \gg$.

7 Le futur cardinal Wiseman était alors supérieur du collège anglais de Rome et agent de l'évêque de Québec auprès de la Propagande. Dans ses Recollections of the four last Popes and of Rome in their times, il écrit, p. 320: "It was not uncommon for Gregory to hesitate in giving his assent to have the papers in the cause brought to himself, and finally to come to a different decision from that of the Congregation. Cardinal Acton used to say that he had known as many as eight or ten items in which the Pope had refused to ratify the judgment of a Congregation, and he had at length reversed it upon canonical grounds which had been overlooked by the many learned persons previously engaged in its discussion. One instance related to Canada. 》

8 Prop. Lettere, 1833, 255-256: 《Rebus omnibus tamen mature perpensis, Sanctitas Sua jussit tibi nomine suo significari se optare A. T. in gerendo memorato munere permanere. $\gg$ 
Mgr Lartigue se soumit. Mais en 1834, il est de nouveau abattu. Par l'entremise de M. Thomas Maguire, député de l'évêque de Québec à Rome, il présente de nouveau sa démission et suggère comme successeur $M$. Antoine Tabeau, curé de Boucherville et vicaire général ${ }^{9}$. Cette fois, la Propagande fut plus prudente. Elle refusa d'accepter la démission de Mgr Lartigue et elle lui donna l'homme de son choix comme coadjuteur et successeur éventuel ${ }^{10}$. De plus, permission était accordée à $\mathrm{Mgr}$ Signay de sacrer M. Tabeau, évêque de Spiga in partibus, dès qu'il le jugerait opportun. Si on avait cru régler le problème, on était loin de compte. Car M. Tabeau qui est malade, décline l'épiscopat ${ }^{11}$; il résiste aux exhortations de Mgr Signay et de Mgr Lartigue ${ }^{12}$. Le 7 mars 1835, Grégoire XVI lui enjoint de se soumettre ${ }^{13}$. Mais il meurt, le 7 mai, avant d'avoir reçu la consécration épiscopale. Ainsi les efforts tentés par Mgr Lartigue pour se démettre et se trouver un successeur aboutissent à un échec. Il faut en dire autant des démarches entreprises pour ériger le district de Montréal en un diocèse distinct et indépendant de Québec ${ }^{14}$. L'opposition du Ministère des Colonies était ici le gros obstacle. Pour aplanir les difficultés, il aurait fallu des négociations entre Londres et Rome; mais ces deux puissances s'ignoraient mutuellement. En 1832, Québec avait suggéré comme intermédiaire un sujet anglais résidant à Rome, le cardinal Thomas Weld ${ }^{15}$. Mais celui-ci s'était dérobé,

\footnotetext{
${ }^{9} \mathrm{RAPQ}$ (1936-37), 266. M. Thomas Maguire à Mgr Maï, Rome 31 juillet 1834 .

10 Ibid., 285. Mgr Maï à Mgr Signay, Rome, 11 octobre 1834.

$11 \mathrm{Ibid}$., 301. Mgr Signay à M. Tabeau. Québec, 16 décembre 1834.

12 Ibid., 303-304. Mgr Signay à Mgr Lartigue, 28 décembre 1834. Mgr Signay à M. Tabeau, 29 décembre 1834 .

13 Ibid., 316. Le cardinal Fransoni à Mgr Signay et à M. Tabeau, Rome, 7 mars 1835.

14 En 1825, le gouverneur Dalhousie avait été pressenti. Il aurait volontiers recommandé l'érection du diocèse de Montréal, si Louis-Joseph Papineau et Denis-Benjamin Viger, cousins de Mgr Lartigue et chefs du parti populaire, s'étaient ralliés à la politique du gouverneur dans la Chambre d'assemblée. RAPQ (1941-1942), 485. Mgr Lartigue à Mgr Poynter, 19 juillet 1826. - En 1829, une requête de Mgr Panet et la recommandation du gouverneur Kempt avaient lamentablement échoué au ministère des colonies. RAPQ (1934-1935), 348. MM. Maguire et Tabeau à Mgr Signay, 4 août 1829. Lettre datée de Londres.

15 RAPQ (1937-38), 31-32. Mgr Turgeon à Mgr Lartigue, 7 juillet 1835. Il avait "suggéré que l'on priât la cour de Rome de tenter elle-même un arrangement par l'intermédiaire du cardinal Weld ».
} 
sûr qu'il était de courir à un échec ${ }^{16}$. D'autre part, le secrétaire de la Propagande, Mgr Maï, déclarait que Rome ne prendrait pas l'initiative des pourparlers ${ }^{17}$. Telle était la situation, la désespérante situation, à l'été de 1835 . Un an plus tard, l'impossible est devenu une réalité. Comment cela ? L'histoire vaut d'être racontée.

\section{MONTREAAL SOUS LE SIGNE DE L'UNITÉ ET DE LA CHARITÉ (septembre 1835)}

M. Tabeau a-t-il offert sa vie pour le rétablissement de la paix à Montréal ? La chose n'est pas certaine; mais on serait porté à le croire, en constatant le rapide et total renversement de la situation qui suivit de près sa mort ${ }^{18}$. Deux manifestations de ce nouvel esprit méritent surtout de retenir notre attention.

\section{LES NOCES D'OR DE M. JACQUES ROQUE, P.S.S.}

En 1860, le docteur Jean-Baptiste Meilleur, premier surintendant de l'Instruction publique dans le Bas-Canada, publiait son Mémorial de l'Education; effort louable pour l'époque et qui n'a pas perdu sa valeur. Il reconnaissait aux individus comme aux institutions la part de mérite qui leur revenait dans le progrès de l'éducation. A sa notice sur les Sulpiciens et sur le Collège de Montréal, il ajoutait en «addendum », un fait sur le sens exact duquel il se méprenait un peu, croyons-nous. Nous

${ }^{16}$ RAPQ (1936-37), 236. M. Thomas Maguire à Mgr Signay, Rome, 30 janvier 1834. Le cardinal Weld lui a dit que le gouvernement britannique s'opposait absolument à ce que le district de Montréal fût érigé en évêché régulier.

17 RAPQ (1936-37), 241. M. Thomas Maguire à Mgr Signay, Rome, 24-26 février 1834. Il vient de voir Mgr Maï. Celui-ci est aussi d'opinion que Rome ne peut négocier avec l'Angleterre au sujet de l'érection de Montréal en évêché.

18 Dans sa lettre du 25 novembre 1835 au préfet de la Propagande, Mgr Lartigue fait un rapprochement entre la mort de M. Tabeau et la détente qui suivit: «Depuis la douloureuse mort de l'Illustrissime et Révérendissime Antoine Tabeau, que la libéralité du Saint-Siège m'avait donné comme coadjuteur dans le gouvernement spirituel du district de Montréal, le Séminaire de Saint-Sulpice de cette ville manifeste plus de sympathie pour ma personne et ma dignité épiscopale; tout le monde en est édifié. AAM, Lettres de Mgr Lartigue, 8, 42. Résumé du document dans RAPQ $(1943-44), 326$. 
lui laissons la parole. Son style solennel, lyrique - c'était le style de l'époque - et un peu embarrassé, nous fera sourire; il n'enlèvera rien à notre joie profonde:

Jeudi, le 24 septembre 1835, eut lieu dans la vaste église de Montréal, la plus belle cérémonie dont cette ville eut jamais été témoin. Suivant les avis depuis longtemps publiés dans les journaux publics, le vénérable Messire Roque, vicaire-général, et pendant 22 ans directeur du Collège de Montréal, célébrait sa cinquantième année de prêtrise. Ses nombreux élèves, environ 500, se réunirent à neuf heures du matin aux bureaux de la fabrique, et de là partirent ayant en tête la musique (bande) du $32^{\mathrm{e}}$ régiment pour aller prendre le clergé au Séminaire de Saint-Sulpice. Le vénérable $M$. Roque, revêtu de magnifiques ornements, marchait assisté de deux prêtres, puis Mgr Lartigue, mitre en tête, la crosse à la main, et assisté de trois prêtres, était suivi du reste du clergé, composé de plus de cent prêtres revêtus de surplis. Jamais auparavant la ville de Montréal n'avait vu un clergé aussi nombreux réuni pour aucune cérémonie quelconque.

Mgr l'évêque, «assistant-paré », la gran'messe fut célébrée avec toute la pompe possible par $M$. Roque, dont l'émotion était bien visible à la foule présente. Lorsque le vénérable prêtre à cheveux parfaitement blancs, et courbé sous le poids des années, vint se jeter aux genoux de l'évêque pour renouveler les vœux de sa prêtrise, tout le monde était attendri ... ${ }^{19}$. Messire Joseph Aubry, membre du Séminaire de Québec, prononça un discours parfaitement approprié à la circonstance, et qui fit sur son auditoire distingué une profonde impression. On estimait à 8000 les personnes présentes à cette imposante cérémonie dont le motif était de satisfaire au sentiment d'une respectueuse et vive gratitude.

Le pain bénit présenté par les élèves de $M$. Roque était d'une hauteur prodigieuse et très bien exécuté ; une charpente de bois avait été faite exprès pour le recevoir et l'exposer dans toute sa beauté aux yeux des assistants. Il était entouré de six colonnes et surmonté d'une colonne impériale; à chaque

${ }^{19}$ Les points de suspension sont du Dr Meilleur. 
colonne pendait un ruban porté par un élève de $M$. Roque, et chaque porteur était choisi de manière à représenter l'une des professions, savoir:

Avocat: Toussaint Pelletier.

Médecin: Dr P. A. Lusignan.

Notaire: P. Lukin.

Arpenteur: André Truteau.

Commerçant: J. A. Donegani.

Ouvrier: L. Paschal Comte.

Il y avait, en outre, six bannières placées autour du pain bénit qui portaient les inscriptions relatives aux différentes professions exercées par les porteurs de rubans.

Le pain bénit, ainsi décoré et accompagné, fut présenté par Benj. Rollin, avocat, le plus âgé des élèves de M. Roque.

Pendant que la foule était sous l'influence du prestige de tant de munificences à la fois significatives et édifiantes, on fit une productive collecte dont le montant fut employé plus tard à des œuvres pies. C'est ainsi que, sous les auspices de la Religion, les joies innocentes du peuple sont converties en actes de charité et de bienfaisance substantielle.

Après la messe eut lieu le chant solennel du Te Deum, avec tout le transport que ce chant sacré provoque dans des cœurs honnêtes mus par la reconnaissance.

Enlevés par la sublimité de ce chant d'actions de grâces que l'Eglise entonne à la suite d'événements heureux, ceux qui y prennent part, pleins d'une sainte allégresse, sont autant d'Ambroise et d'Augustin qui s'adressent à Dieu pour le remercier d'une insigne faveur reçue, et l'on peut dire que celle qu'eurent les élèves de M. Roque, de pouvoir se réunir, pour fêter avec lui sa cinquantième, étant une des grandes fêtes de leur vie, ils prirent part simultanément à ce chant des saints avec une spontanéité et un enthousiasme qu'il serait impossible de décrire.

Voilà pour ce qui se passe à l'intérieur de l'église; mais, au dehors, la poésie n'étant pas indifférente au sentiment qui animait la fête, voulut y contribuer aussi, en jetant son bouquet de fleurs, composé pour la circonstance par Pierre Laviolette, sujet marquant parmi les élèves de M. Roque. 
Après le Te Deum, les élèves reconduisirent $\mathrm{M}$. Roque au Séminaire de St-Sulpice, où M. Toussaint Pelletier fit une harangue, au nom des élèves et lui présenta les six bannières que portaient les membres des professions. M. Roque parut très affecté de ces marques de respect et d'attachement de la part de ses élèves; mais ils remplissaient avec bonheur un devoir dont j'aurais été extrêmement heureux de partager avec eux l'accomplissement. Des circonstances impérieuses m'empêchèrent de le faire, et j'ai toujours regretté depuis, d'avoir été privé de cette douce consolation. Aujourd'hui au moins, je suis heureux de pouvoir combler un peu cette lacune dans ma vie, contrariée par tant de causes diverses. Je puis le faire au moyen de cette imparfaite note dont un ami a eu la bonté de me procurer les matériaux à ma demande pour cette fin ${ }^{20}$.

Le docteur Meilleur a-t-il bien compris tout le sens de cette fête, dont il rappelait le souvenir vingt-cinq ans après l'événement? Il a voulu manifester sa reconnaissance à ses anciens maîtres; et celle-ci entre bien pour quelque chose dans la manifestation du 24 septembre 1835. Mais il y a plus. Le jubilé d'or d'un prêtre est ordinairement une fête de famille, réservée aux intimes du clergé et aux plus proches parents. En temps normal, combien des 500 élèves de $M$. Roque se seraient rappelé, combien se seraient fait un devoir d'assister à l'événement ? Aujourd'hui, ils sont tous là, à de rares exceptions près; et ils ne sont pas seuls : 8,000 personnes, un jour ouvrier, le jeudi, 24 septembre 1835. Nous le répétons: le peuple chrétien en général, au Canada comme ailleurs, ignore le sens profond d'une pareille manifestation. Mais aujourd'hui, à Montréal, tout le monde en comprend le sens particulier: car c'est un Sulpicien, M. Jacques Roque, qui renouvelle les vœux de sa prêtrise devant Mgr Jean-Jacques Lartigue, évêque de Telmesse.

Le peuple canadien tenait de ses origines françaises la passion des belles fêtes et des grands déploiements, le besoin de manifester au grand jour ses sentiments de joie. Depuis 15 ans, il vit à Montréal, privé de ce plaisir, replié sur lui-même. Il

20 Mémorial de l'Education, 63 et ss. 
veut d'un seul coup reprendre tout le temps perdu: un pain bénit comme il n'y en eut probablement jamais dans l'histoire du Canada; une collecte improvisée, semble-t-il, car on en employa plus tard seulement le revenu au profit des œuvres pies, mais elle n'en fut pas moins «productive»; cent prêtres, presque tout le clergé du district, passant, fanfare en tête, du Séminaire à la Paroisse; à la sortie de l'église, un élève devenu poète pour la circonstance, dédie ses vers à M. Roque; un ancien lui fait une «harangue».

N'avons-nous pas raison de le dire ? A l'occasion du jubilé d'or de M. Roque, on célèbre l'union des esprits et des cœurs depuis longtemps perdue, toujours attendue, enfin retrouvée. On voit des amis, des frères là où l'on s'était habitué à voir des traîtres, des ennemis. Hier encore, on s'épiait, on se surveillait; aujourd'hui, on s'aime, on s'admire, on collabore. Il n'y a plus qu'un seul troupeau sous un seul Pasteur, le vrai. Quam bonum et quam jucundum habitare fratres in unum!

\section{LA REQUETE DU CLERGE}

Cette union des esprits et des cœurs parait durable, constructive. Elle est du même mois de septembre 1835 la requête, rédigée par M. Quiblier, supérieur du Séminaire, portant 129 signatures, tout le clergé du district, et adressée à Sa Sainteté Grégoire XVI ${ }^{21}$. Que demande-t-elle ? L'érection du district de Montréal en un diocèse distinct, relevant immédiatement de Rome. Et quelles sont les raisons mises en avant ? Celles-là mêmes que Mgr Lartigue ne cesse de répéter depuis 15 ans. L'autorité insuffisante du suffragant; l'importance du district: d'un côté 400 milles d'étendue, de l'autre, les limites sont encore indéfinies; il est populeux, 96 paroisses, des missions naissantes, en tout 230,000 catholiques. L'évêque trouvera facilement les revenus dont il aura besoin; l'assentiment de l'autorité civile peut être considéré comme certain. Enfin, on obtiendra par là cette concorde et cette paix depuis si longtemps souhaitées par Sa Sainteté. Et qui désire-t-on pour premier évêque de Montréal ? Nul autre que $\mathrm{Mgr}$ Jean-Jacques Lartigue, évêque de

\footnotetext{
${ }^{21}$ RAPQ (1943-44), septembre 1835.
} 
Telmesse. Qui oserait dire, qui oserait penser qu'une telle requête n'est pas promise au succès ? Et pourtant, la partie n'est pas encore gagnée, loin de là.

\section{L'ATTITUDE DE MGR SIGNAY}

Ce n'était pas à Mgr Lartigue, simple suffragant, mais bien à l'évêque diocésain, $\mathrm{Mgr}$ Joseph Signay, qu'il appartenait d'acheminer la requête vers Rome ${ }^{22}$. Or cette nécessaire intervention de l'évêque de Québec allait compliquer et retarder, au lieu d'accélérer la solution du problème. Sur l'opportunité d'ériger le diocèse de Montréal, Mgr Signay et son coadjuteur, Mgr Turgeon, ne pensaient pas autrement que Mgr Lartigue. Mais, sur les moyens d'arriver au but, le désaccord était complet. Fidèle à la conception de ses précécesseurs en des cas analogues ${ }^{23}$, Mgr Signay croyait inutile, voire dangereux, de demander l'assentiment de Rome avant d'avoir obtenu d'abord celui de Londres. «A Londres d'abord, à Rome ensuite », telle était l'attitude de Québec ${ }^{24}$. Et c'est pourquoi Mgr Signay retient la requête à Québec au lieu de l'adresser immédiatement à Rome.

22 En transmettant la requête à l'évêque de Québec, les pétitionnaires écrivent: «Notre démarche sera agréable au St-Siège, comme nous savons qu'elle l'est à Votre Grandeur, si vous avez la bonté de l'appuyer de votre puissante recommandation. 》 Ibid. - L'accusé de réception de Mgr Signay est du 20 octobre. Rien ne pouvait entrer davantage dans mes vues que cette supplique, écrit-il, «que je vais m'empresser de faire déposer aux pieds du Vicaire de Jésus-Christ et supplier Sa Sainteté de vouloir bien avoir égard aux vœux qui $\mathrm{y}$ sont exprimés 》. RAPQ (1943-44), 315.

23 Quand en $1819, \mathrm{Mgr}$ Plessis voulut diviser son vaste diocèse, il se rendit d'abord à Londres et accepta tous les refus du ministre des colonies, Bathurst, avant de proposer le problème à Rome. Mgr Bourget et son Temps, I: 24 et ss. - En 1829, MM. Thomas Maguire et Antoine Tabeau sont délégués en Europe pour défendre et promouvoir les intérêts du diocèse de Québec. Ils se rendent d'abord à Londres; et conformément à leurs instructions, ils soumettent au ministre le projet d'un diocèse catholique à Montréal. Le refus de sir George Murray fut catégorique; et ce refus leur interdisait de poser le problème à Rome. Nous avons précisément sur ce sujet un texte de Mgr Signay. Il écrit à Mgr Lartigue le 17 décembre 1834 . $\mathrm{Si}$ autrefois, MM. Maguire et Tabeau «n'ont pas fait cette demande au S. Siège, c'est qu'ils avaient échoué dans une pareille demande à la cour de $\mathbf{S}$. James, dont il fallait obtenir l'assentiment avant de faire aucune démarche à Rome 》. RAPQ (1936-37), 179.

24 Disons-le une fois pour toutes: l'attitude des évêques de Québec n'était pas le résultat d'une erreur doctrinale. Ils connaissaient et ils admettaient le droit premier et exclusif du Saint-Siège dans l'érection des diocè- 
L'attitude de Montréal était tout autre et peut se formuler ainsi : "A Rome d'abord, à Londres ensuite ». Dès 1831, Mgr Lartigue avait écrit que «notre gouvernement reconnaîtrait [le diocèse de Montréal] comme il a fini par reconnaître nos paroisses quand il a vu qu'elles existaient de fait et qu'on pouvait les ériger sans lui ${ }^{25}$. $\gg$ Quatre ans plus tard, c'est-à-dire au moment où Mgr Signay retient indûment à Québec la requête du clergé de Montréal, l'évêque de Telmesse écrit:

Avec un gouvernement protestant, on doit s'atendre qu'il ne vous donnera jamais, formellement du moins, les nouvelles choses qui deviendront de temps à autre nécessaires pour la Religion; mais nous n'avons qu'à les prendre nous-mêmes, et ensuite, il les agréera ${ }^{26}$.

Et n'est-ce pas la leçon qui se dégage de notre propre histoire religieuse ? Si Mgr Briand avait attendu une permission écrite de Londres avant de se faire sacrer et de prendre possession du siège de Québec en 1766 , où en serait aujourd'hui l'Eglise du Canada? Il n'a pas craint d'assumer le risque; et dans la suite ses successeurs ont été reconnus par Londres. De même, si douloureuse qu'elle ait été pour lui et si imparfaite dans ses effets, la présence de Mgr Lartigue à Montréal depuis 1821 n'en est pas moins un fait que le gouvernement ne pourra pas ignorer indéfiniment et qui hâtera la reconnaissance civile du diocèse. Mais si l'évêque de Telmesse avait attendu la permission écrite du Ministre des colonies pour exercer sa juridiction, construire son église et sa résidence, tout serait à faire aujourd'hui. Les beaux et solides raisonnements de Mgr Lartigue laissaient inentamée «l'attitude de Québec». Et pour cause, comme nous allons le voir.

ses. Leur attitude était prudentielle; et elle s'explique - si elle ne se justifie pas toujours - par le fait que la liberté de l'Eglise du Canada n'étant pas suffisamment garantie par des documents écrits, était, en quelque sorte, à la merci du ministre des colonies. Et l'on craignait, en allant trop vite ou en demandant trop, d'indisposer le ministre et de nuire à l'équilibre actuel des relations entre l'Eglise et l'Etat.

25 RAPQ (1942-43), 114-115. Mgr Lartigue à Mgr Panet, 8 sept. 1831.

26 RAPQ (1943-44), 328. A Mgr Turgeon, 30 novembre 1835. Les soulignés sont de nous. 


\section{LE PROJET DE MONTREAL SOUMIS À LA COMMISSION GOSFORD}

Conséquence des 92 résolutions de 1834 et de la crise politique où était plongé le Bas-Canada, le gouvernement impérial rappelait Aylmer à Londres et nous donnait comme gouverneur général lord Gosford. Celui-ci était en même temps haut-commissaire: avec ses adjoints, sir Charles Grey et sir George Gripps, il était chargé « de faire une enquête complète et impartiale sur toutes les plaintes qui leur seraient soumises au sujet de l'administration et du gouvernement de la province ${ }^{27}$.» Arrivé à Québec le 23 août 1835, Gosford posa des gestes qui furent interprétés comme une condamnation de la politique de son prédécesseur Aylmer et comme la volonté de rendre enfin justice aux Canadiens français. «Il invita M. Papineau et M. Viger à dîner au Château, écrit Chapais. Il voulut faire un compliment à notre clergé en allant visiter les classes du Séminaire. Sa courtoisie, son affabilité, son désir de plaire produisirent leur effet, et au moment de l'ouverture de la session [27 octobre], il semblait y avoir une détente dans la situation ${ }^{28}$. Mgr Signay est au nombre de ceux qui voient en Gosford le sauveur si longtemps attendu. Il écrit à Mgr Lartigue:

Ce personnage, animé du désir de rendre justice, m'a déjà témoigné des sentiments propres à me faire espérer une heureuse issue de mes dernières démarches auprès de lui, dans l'affaire du Sault-SaintLouis ${ }^{29}$.

L'atmosphère d'euphorie qui régnait à Québec nous aide à comprendre le geste que posa alors Mgr Signay, et qui après plus d'un siècle nous apparaît encore comme un faux pas: à l'insu de Mgr Lartigue, il soumettait à la Commission royale d'enquête le projet du futur diocèse de Montréal ${ }^{30}$. La mau-

27 Chapais, Cours d'Histoire du Canada, IV : 56.

28 Ibid.: 59.

29 RAPQ (1937-38), 54, 13 octobre 1835.

30 Ibid., 60. Walcott, secrétaire civil à Mgr Signay, Québec, 31 octobre 1835. Il accuse réception, au nom du gouverneur, de la lettre de $\mathrm{Mgr}$ l'évêque de Québec au sujet de l'érection du district de Montréal en un évêché séparé de celui de Québec. 
vaise humeur de Mgr Lartigue est à son comble. Il écrit à Mgr Signay:

Je pensais bien qu'après vos premières objections contre la requête du clergé de Montréal, que vous vous étiez engagé à envoyer et à appuyer auprès du Saint-Siège, vous en trouveriez d'autres dans vos rapports avec le Gouvernement, comme si celui-ci devait régler notre Eglise et pourvoir à ses besoins. Et d'abord, pourquoi s'adresser avant tout au Gouvernement, afin de se procurer des entraves? On ne l'avait pas fait pour l'épiscopat de M. Tabeau; le clergé ne l'avait pas demandé, et même l'évêque de Québec avait promis de dire au Gouvernement que cette division était nécessaire, ce que souhaitait aussi le coadjuteur ... Pour moi, je me perds en toutes les conjectures que suggèrent ces marches et contremarches; mais je souhaite que le clergé de mon district prenne ces incertitudes ou délais en bonne part; et qu'il ne les regarde pas comme un déni de ce qu'on lui avait formellement promis ${ }^{31}$.

Et à Mgr Turgeon:

Je trouve, sauf meilleur avis, qu'on a eu tort de s'adresser à la Commission royale pour l'affaire de l'érection de Montréal en évêché; car elle n'est venue que pour recueillir les griefs des deux partis politiques; or cette affaire d'érection ne peut assurément passer pour un grief; elle [la Commission] ne décidera rien par elle-même ${ }^{32}$.

Le problème, en effet, n'était pas de la compétence des commissaires; et on ne voit pas qu'ils s'en soient occupés ${ }^{33}$. Mais puisqu'il leur était référé, force était à Mgr Signay d'attendre leur réponse avant de tenter toute autre démarche. Il proteste qu'advenant un refus, il s'adressera à Rome. Mais, en attendant, il retient précieusement à Québec la requête du clergé

${ }^{31}$ RAPQ (1943-44), 319, 7 novembre 1835.

32 Ibid., 322,19 novembre 1835.

33 Dès le 20 novembre 1835, un des commissaires, sir Charles Grey, avait déclaré que le problème n'était pas de la compétence de la commission et qu'il serait référé à Londres. RAPQ (1943-44), 323-324. Mgr Lartigue à Mgr Signay, 20 novembre 1835. - D'après une lettre de M. Pierre Brunet, archiviste national adjoint, à l'auteur, en date du 15 février 1962, " un examen attentif du rapport de la commission Gosford n'a révélé aucune déclaration concernant l'érection du diocèse de Montréal ». 
de Montréal et il s'enferme lui-même dans un mutisme complet. Le 2 novembre, il adresse une longue dépêche au préfet de la Propagande ${ }^{34}$; pas un mot du problème de Montréal. Le 18, nouvelle lettre au cardinal Fransoni et qui sera remise à celui-ci par Mgr Provencher. Le suffragant de la Rivière-Rouge est constitué procureur de l'évêque de Québec auprès de la Propagande; les buts précis de son voyage sont énumérés, mais aucune mention explicite n'est faite du projet de Montréal ${ }^{35}$. Les plus optimistes, tel Mgr Turgeon, exprimaient l'avis que la Commission se montrerait favorable à l'érection du diocèse de Montréal. Et quand il en serait ainsi, combien de temps faudra-t-il attendre ? Car l'enquête royale n'est pas au terme de ses travaux, elle vient de les commencer. Et puis, les commissaires ne peuvent émettre qu'une opinion, la décision finale appartenant toujours au Ministre des colonies. Plus on avance dans le temps, plus la solution désirée paraît s'éloigner. C'en était décidément trop pour la patience des Montréalais. Mais comment sortir de l'impasse?

\section{SUPREME DÉMARCHE DU CLERGÊ DE MONTRÉAL}

Devant les «marches et contremarches» de Québec, le clergé de Montréal décide de s'adresser directement au SaintSiège en passant par dessus la tête de Mgr Signay. MM. Quiblier, supérieur du Séminaire, et Hyacinthe Hudon, v.g., prennent la direction du mouvement. La requête de septembre est de nouveau signée par tous les prêtres du district et adressée au secrétaire de la Propagande. La lettre qui l'accompagne raconte l'attitude de Mgr Signay, mais sans juger les intentions. En transmettant la requête au Saint-Siège, écrivent MM. Quiblier et Hudon, nous voulons uniquement le bien de l'Église du Canada; et nous avons la certitude de rendre service à Mgr Signay lui-même en le tirant du faux pas dans lequel il s'est malheureusement engagé. Car il acceptera la décision de Rome; il en sera soulagé et heureux ${ }^{36}$.

34 RAPQ (1937-38), 60.

35 Ibid., 64.

36 RAPQ (1943-44), 324-325. MM. Quiblier et Hudon à Mgr Maĩ, Montréal, 21 novembre 1835. 
Cette suprême démarche du clergé de Montréal allait avoir les conséquences les plus heureuses. Mgr Signay ne pouvait plus garder le silence vis-à-vis de Rome, puisque la requête qu'il avait retenue à Québec serait bientôt parvenue au Saint-Siège. Il savait de plus que, si Mgr Provencher ne plaidait pas la cause de Montréal comme procureur de l'évêque de Québec, il le ferait en son nom personnel. Ces considérations l'amenèrent à modifier son attitude: sans attendre davantage la réponse de la Commission royale d'enquête, il acheminait la requête de Montréal vers Rome et il chargeait Mgr Provencher d'en être le champion devant la Propagande. ${ }^{36 a}$

Cette attitude nouvelle de Mgr Signay ne pouvait laisser Gosford indifférent. Le gouverneur avait tout lieu de croire que Rome, exauçant le vœu unanime des évêques, érigerait le diocèse de Montréal; et cela sans entente préalable avec Londres. Or, si par respect pour l'intangible loi de suprématie, le gouvernement impérial refusait de reconnaître la décision de Rome, qu'arriverait-il ? Président de la Commission Royale, Gosford connaissait les nombreux griefs du Bas-Canada contre Londres. L'heure était décidément mal choisie pour en ajouter un autre, et de belle taille, en mécontentant les évêques, le clergé, les catholiques, c'est-à-dire l'immense majorité de la province. De plus, indépendamment des conséquences politiques, Gosford avait l'âme assez haute, il avait assez le sens de la justice pour reconnaître le bien-fondé de la requête de Montréal. Pour toutes ces raisons, un refus de Londres lui paraissait impossible, impensable dans les circonstances. Il appartiendra au ministre des colonies, pense-t-il, de trouver un moyen de concilier le droit intangible avec le fait inéluctable. Quant à lui, il obéira à la dictée de sa conscience; il recommandera la reconnaissance par Londres du diocèse catholique de Montréal. Mais avant d'adresser sa dépêche au ministre, il aura un ultime entretien avec l'évêque de Québec. Laissons ici la parole à Mgr Signay:

36a RAPQ (1937-38), 77. Mgr Signay à Mgr Provencher à Londres. Québec, 11 janvier 1836. Mgr Provencher ne devra pas «laisser ignorer à la Propagande que le gouvernement n'a pas encore donné son assentiment à la division du diocèse ». Mais il pourra « dire qu'on a lieu d'espérer qu'il y consentira ». 
Lord Gosford m'a demandé une entrevue et précisément sur la division des districts. Je l'ai trouvé tout à fait disposé à recommander la mesure, ce qu'il ne voulait faire, m'a-t-il dit, sans avoir une parfaite connaissance de toutes les correspondances qui ont eu lieu sur cette affaire. Je n'ai pas oublié le petit paragraphe de votre lettre sur le sujet ${ }^{37}$. Il se flatte beaucoup du succès, espérant une réponse en mai. Qui empêcherait Mgr Provencher de négocier en notre nom ? ${ }^{38}$, car Lord Gosford lui-même trouverait cela avantageux, d'autant plus que Son Excellence a spécialement recommandé ce prélat à Lord Glenelg. Tout ceci convaincra qu'en allant avec une certaine modération, et non brusquement,

37 Il s'agit, croyons-nous, de la phrase suivante de la lettre de Mgr Lartigue à Mgr Signay, 3 février 1836: «Quant au Gouvernement et à la Commission, qui, comme on me le marque, n'ont montré de scrupule pour reconnaître un évêque de Montréal, que celui d'avoir l'air d'approuver un acte de N. S. P. le Pape, qu'en bons Protestants, ils ne peuvent agréer, il me semble très facile de lever ce scrupule et de pacifier leur conscience; qu'ils ignorent parfaitement et soient censés ignorer que le Pape ait érigé un diocèse à Montréal ou y ait nommé un évêque en titre; mais qu'ils se contentent de reconnaître pour diocèse et évêque de Montréal celui que l'évêque de Québec reconnaîtra et qualifiera comme tel. Qu'ils regardent l'évêque de Québec comme le seul supérieur de l'Eglise catholique en ce pays; mais alors qu'ils regardent aussi chaque chose ou personne religieuse comme il les qualifie. Je les défie de répondre rien de raisonnable contre cette proposition, si on les pousse fortement là-dessus. 》 AAM., Lettres de Mgr Lartigue, 8, 179. La pensée n'est pas d'une intelligence facile, il faut le reconnaître. Mais RAPQ (1944-45), 114, ne rend pas justice à Mgr Lartigue quand il résume: «On tranquilliserait les protestants en leur suggérant de reconnaître l'évếque de Québec au lieu du Pape, comme le seul Supérieur de l'Eglise catholique en ce pays pour ce qui regarde l'érection d'un diocèse à Montréal. 》 La pensée de Mgr Lartigue n'a rien de schismatique. Il n'a jamais eu l'intention de «suggérer aux protestants de reconnaître Mgr Signay au lieu du Pape, comme seul Supérieur de l'Eglise du Canada ». Mais étant donné qu'ils peuvent, en toute tranquillité de conscience, ignorer délibérément le Pape d'une part, et, d'autre part, reconnaître expressément Mgr Signay comme seul supérieur de l'Eglise en ce pays, il agiront conformément à leur conscience en conformant leur conduite à celle de Mgr Signay dans le cas particulier de Montréal.

38 Avant même de recevoir cette délégation, $\mathrm{Mgr}$ Provencher avait plaidé devant le ministre des colonies la cause de Montréal. De Londres, il écrit à Mgr Signay, le 19 janvier: «Ici j'ai parlé à Lord Glenelg au sujet de l'évêché de Montréal. J'ai été reçu poliment, et il m'a dit qu'il examinerait cette question et qu'il me répondrait. Il paraissait en avoir la volonté en prenant mon adresse. Je lui ai dit alors le temps de mon départ. Je suis retourné une autre fois, et il était allé à la cour. Son frère, qui était secrétaire, m'a dit: "il reviendra ce soir et vous aurez votre réponse demain ». Je crois qu'elle viendra demain pendant longtemps. » Bull. Soc. Hist. de Saint-Boniface, III : 147-149. 
comme on voulait me faire marcher, les choses se feront à souhait; mais que l'on me donne un peu de temps. Au reste, envoyez-moi vos remarques sur la marche à continuer. Car le bon lord est tout à fait aimable, et je crois sincèrement qu'il y va de franc jeu. J'ai eu samedi une longue entrevue tout entière sur le sujet, et je lui ai donné diverses connaissances qui ont paru le satisfaire. Il paraît même tenir à honneur de voir s'opérer l'érection désirée sous son administration. Il faut en profiter d'autant plus qu'il est en rapports très intimes avec Lord Glenelg [Ministre des colonies] ${ }^{39}$.

Pour ce qui regarde l'assentiment encore à venir de Londres, cet entretien de Mgr Signay avec lord Gosford est d'un intérêt capital; et il est juste de reconnaître que l'évêque de Québec a très efficacement servi la cause. Les « certaines connaissances qu'il a données à lord Gosford et qui ont paru le satisfaire » constituent, croyons-nous, la base essentielle du mémoire annexé par Gosford à sa dépêche du 9 février 1836. Dans la dépêche elle-même, fort brève, le gouverneur se dit favorable au projet de Montréal; l'évêque de Québec, ajoute-t-il, connaît mon sentiment; il sait également qu'il n'est pas dans mes attributions de régler définitivement la question. Si donc le ministre répugne à innover, il pourra consulter ses archives et apprendre là quelle ligne de conduite fut tenue lors de l'érection du diocèse de Kingston.

Le Mémoire qui accompagne la dépêche a toutes les allures d'un plaidoyer en règle, et il peut se ramener à ces trois points:

1. Les raisons apportées par l'évêque de Québec: l'accroissement considérable de la population. Il résulte de là que le suffragant de Montréal, n'étant pas reconnu par l'État, ne peut pas rendre à l'évêque de Québec tous les services que celui-ci serait en droit d'en attendre. Le refus de Londres ajouterait au malaise de la province. Comment faire admettre au peuple que «le libre exercice de la religion» ne comporte pas le droit de diviser un diocèse devenu trop considérable pour être administré par un seul évêque?

${ }^{39}$ RAPQ (1937-38), 83. A Mgr Lartigue, 8 février 1836. 
2. Gosford signale ensuite - mais le dossier n'est pas complet - les pièces que possèdent ses archives sur le sujet. Il constate que de 1817 à 1827, l'attitude de Bathurst, alors ministre des colonies, a évolué de l'intransigeance absolue à une sympathie réelle pour l'érection d'un diocèse catholique à Montréal. Deux des prédécesseurs de Gosford, Dalhousie en 1825 et Kempt en 1829 , ne voyaient pas d'objection au projet.

3. Abordant enfin le problème, tel qu'il se pose en 1836 , Gosford en considère la légitimité et l'opportunité. D'après les juristes, le gouvernement impérial ne peut reconnaître un évêque catholique au Canada sans violer la loi de suprématie, qui fait du Roi le chef spirituel suprême dans toute l'étendue de l'Empire. La difficulté est tout entière d'ordre légal et technique. En fait, le gouvernement impérial a plus d'une fois ignoré délibérément un statut qui date de 250 ans, qui n'a plus sa raison d'être et qui ne s'appliquait qu'à l'Angleterre proprement dite. Dans les colonies lointaines, dont plusieurs sont habitées presque exclusivement par des catholiques, on n'a jamais invoqué la loi de suprématie, on a même cherché et trouvé des moyens de l'éluder. N'a-t-on pas vu en ces dernières années des évêques catholiques à Trinidad (1820), à Grenadine ${ }^{40}$, à Terre-Neuve ${ }^{41}$ ? Et tout cela ne s'est pas fait à l'insu du gouvernement. Comment expliquer que l'antique diocèse de Québec ait donné naissance aux diocèses de Kingston (1826) et de Charlottetown (1829) ? Si la loi de suprématie devait s'appliquer envers et contre tout, comment expliquer que depuis 1766, il y ait toujours eu un évêque catholique à Québec ?

Quant à l'opportunité de diviser le diocèse de Québec, Bathurst l'a reconnue pour le Haut-Canada et pour l'Ile-duPrince-Édouard. En 1825, il était tout disposé à en faire autant pour Montréal. Le procureur général Stuart dit que la chose n'est pas nécessaire; mais il se contente d'affirmer. L'évêque de Québec est d'un avis contraire. Comme il s'agit d'un problème ecclésiastique, il ne serait pas sage de préférer l'ignorance

40 Grenadines: une des Iles-Sous-le-Vent, Antilles anglaises.

41 Terre-Neuve: vicariat apostolique depuis 1796; deviendra diocèse 
de l'homme de loi au témoignage et aux raisons de l'évêque de Québec, résultat de l'expérience. Gosford conclut que les difficultés techniques ne sont pas insurmontables, qu'il serait extrêmement dangereux de rejeter une mesure voulue par le clergé et désirée par les chefs du parti populaire de la province. Et voici le mot de la fin: "Toute la question se résume à un arrangement relatif à la discipline d'une religion, dont l'exercice a été garanti aux habitants du Bas-Canada par le traité de paix et par la loi ${ }^{42} »$.

Ce texte fait vraiment honneur à Gosford. A notre connaissance, aucun gouverneur n'avait manifesté autant de compréhension et de sympathie pour les catholiques du Bas-Canada; aucun n'avait osé souligner les illogismes, pour ne pas dire l'arbitraire, de Londres dans l'application de la loi de suprématie; aucun n'avait osé dire au ministre jusqu'où s'étendait «le libre exercice de la religion », garanti aux catholiques du Bas-Canada «par le traité de paix et par la loi ». L'avenir allait prouver que Gosford était un ami sincère du Bas-Canada français et catholique. Mais quel sera le résultat de sa dépêche du 9 février 1836 ?

\section{LA RÉPONSE DE GLENELG}

Gosford avait espéré une réponse en mai. Il était loin de compte; car le document si impatiemment attendu n'arrivait à Québec qu'à la fin de juillet ${ }^{43}$. Répondait-il du moins à l'attente des parties intéressées ? Pour le bien comprendre, il faut en considérer et la lettre et l'esprit. L'étude des pièces d'archives, suggérée par Gosford ${ }^{44}$, avait révélé que jamais un ministre des colonies n'avait, par un acte de sa main, reconnu l'existence

42 \% The whole matter in question being an arrangement in the Church discipline of a Religion, of which the enjoyment has been guaranteed by the treaty and Statute to the inhabitants of Lower Canada. \ La dépêche de Gosford et le mémoire qui l'accompagne sont aux Archives Publiques du Canada.

43 C'est le 1 er août 1836 que Walcott, secrétaire civil, transmet à Mgr Signay une copie de la dépêche de Glenelg. RAPQ (1937-38), 112.

${ }^{44} \mathrm{La}$ dépêche de Gosford du 9 février porte en marge cette note de Glenelg: $\& M^{\mathrm{r}}$ Murdock will examine what course has been taken in the ten North American Provinces to obviate the legal difficulties with which this question is environed. 》 
d'un évêque catholique au Canada ${ }^{45}$. On avait laissé ce soin au gouverneur local. Mais l'expérience avait suffisamment démontré qu'au Canada l'existence des évêques catholiques était compatible avec le droit écrit anglais ${ }^{46}$. En conséquence, Glenelg n'innovera pas; il suivra la ligne de conduite de ses prédécesseurs; et cela veut dire qu'en temps opportun, Gosford sera autorisé à reconnaître l'évêque de Montréal. Mais par-delà le texte écrit, il y avait un esprit nouveau, plus libéral que dans le passé: l'esprit manifesté par Gosford dans sa dépêche du 9 février et que Glenelg faisait sien: «Je n'ai pas besoin de rappeler à Votre Excellence le désir qu'éprouve le gouvernement de Sa Majesté d'adopter toutes les mesures nécessaires susceptibles de répondre aux vœux de ses sujets catholiques du BasCanada, en tout ce qui concerne leur instruction religieuse ${ }^{47}$. Le gouvernement, ajoute-t-il encore, n'innovera en rien. « Mais en même temps, il désire satisfaire la population catholique sur ce sujet; car elle a l'appui de Votre Excellence et de Mgr Bramston ${ }^{48}$. 》 C'était dire que la pensée de Glenelg allait aussi loin que celle de Gosford et que l'heure était désormais dépassée où la loi de suprématie, droit prétendu intangible, mettait des entraves au progrès de l'Église du Bas-Canada.

\section{DE LA DÊPECCHE DE GLENELG Ã L'EXPÉDITION DES BULLES}

Il était écrit que l'érection du diocèse de Montréal serait, jusqu'à la fin, difficile! Par une lettre de Mgr Provencher, on avait appris, au début de mai, les heureuses décisions prises

\footnotetext{
45 PRO (Londres), 261, 499.

46 Murdock ignore cependant deux documents: 1 . Le chèque adressé en 1813 par Bathurst à Mgr Plessis, évêque catholique de Québec; 2. De même, quand en 1827, Bathurst ordonne à Dalhousie de remettre la gratification annuelle à Mgr Panet, évêque catholique de Québec, il avait bel et bien l'intention de le reconnaître comme tel. RAPQ (1933-34), 337-338. Mgr Poynter à Mgr Panet, 23 février 1827. - Voici la conclusion de Murdock: "It would appear therefore that altho the technical difficulties of the question have precluded the issue of any formal Instrument appointing or recognizing a Roman Catholic Bishop in a British Colony, there has been no practical impediment to their exercising under that Title Episcopal Authority over the clergy of their Church. $\gg$ PRO, loc. cit.

47 RAP (Ottawa, 1931), 386. Aussi RAPQ (1937-1938), 112.

$48 \mathrm{Mgr}$ James Bramston, vicaire apostolique de Londres, 1827-1836.
} 
par la Propagande, le 21 mars. Encore un peu de temps, et le grand désir sera devenu une belle, une consolante réalité. Que faut-il pour cela? Une chose, une seule chose qui ne peut manquer et qui ne saurait tarder: l'arrivée des bulles manifestant la volonté du Saint-Père.

Hélas ! la joie de Montréal allait encore une fois se changer en une amère déception! Comme ils furent longs ces mois de mai, juin, juillet, août 1836 ! Il va sans dire que le clergé de Montréal se crut de nouveau trahi, et par qui ? Par Mgr Signay. C'était celui-ci, c'était lui, à n'en pas douter, qui empêchait la volonté du Pape de réjouir le cœur des Montréalais. Et on ne songeait à rien moins qu'à une seconde suprême démarche auprès du Saint-Siège. Témoin la lettre que le pacifique M. Ignace Bourget, secrétaire de Mgr Lartigue, adressait au non moins pacifique M. C.-F. Cazeau, secrétaire de Mgr Signay, le 26 juillet 1836 :

A coup sûr, je ne voudrais pas vous faire manquer à la confiance qu'a en vous Monseigneur, en trahissant le secret que vous lui devez. Mais si je pouvais être informé au plus tôt de ce qu'a fait Monseigneur de Québec pour lever les obstacles qu'il semble avoir mis à l'expédition des Bulles concernant le district de Montréal, on pourrait probablement arrêter une démarche que projette le clergé du district qui commence à se remuer, voyant que l'affaire de la séparation des deux diocèses tablée à Rome, le 21 mars dernier n'avance plus à rien; et qui se doute que cette marche lente est dirigée par l'Ev. de Québec. Il est plus que probable que Sa Grandeur aurait du déplaisir de voir ainsi le clergé du district le talonner de si près, sans compter plusieurs autres inconvénients qui peuvent résulter de ces différentes pétitions ${ }^{49}$.

A cette date, 26 juillet, les bulles étaient en route pour Montréal, où elles arriveraient le 29 août. Il n'en reste pas moins qu'un intervalle de trois mois s'était écoulé entre la

${ }^{49}$ AAQ. D. M. H., 11. Déjà le 11 juillet, M. Bourget avait adressé à M. Cazeau une semblable lettre restée sans réponse. Ibid. - Mgr Provencher, qui était à Québec, à la fin de juillet, conseilla la patience, et la seconde suprême démarche n'eut pas lieu. 
décision de la Propagande et l'expédition du document lui donnant force de loi, 21 mars-18 juin. Ce n'est pas sans raison qu'après avoir attendu trois mois, le Saint-Père achemine les bulles vers Montréal. Un événement a dû se produire qui a fait tomber les hésitations. Lequel ? Ne serait-ce pas la connaissance, parvenue à Rome, sinon du texte de la dépêche de Glenelg à Gosford, du moins des excellentes dispositions du ministre ? Mais comment cela s'est-il fait ? Et Mgr Signay a-t-il dirigé la «marche lente de Rome», comme l'insinuait M. Bourget?

Deux questions auxquelles nous tenterons d'apporter une réponse. Car l'état actuel de la documentation ne rend pas compte du délai de Rome dans l'expédition des bulles. Serait-ce un effet de ce que l'on appelle «le hasard »? Et puis, est-ce aussi «par hasard» qu'au moment où les bulles arrivent à Montréal l'assentiment de Londres est déjà obtenu ? Faudra-t-il admettre que les hommes n'ont eu aucune part consciente à cette heureuse suite des événements ? Nous ne le pensons pas. L'explication que nous proposons ici ne contredit aucune des connaissances que nous avons par ailleurs. Au lecteur de juger si elle contient quelque élément valable.

Entrons en matière, et commençons par aligner deux faits que nous connaissons avec certitude:

1. Le 24 février 1836, Mgr Signay adressait à la Propagande une dépêche que celle-ci reçut après le 21 mars ${ }^{50}$. Que disait donc l'évêque de Québec ? Il avait eu un entretien avec Gosford, et celui-ci s'était montré très sympathique à l'érection du diocèse de Montréal. Mais il avait eu soin d'ajouter que la décision finale appartenait au ministre des colonies. Dès lors, avait conclu Mgr Signay, un désaccord reste encore possible entre Londres et Rome. Cette seule possibilité émeut profondément l'évêque de Québec. Et, dernière manifestation de ce que nous avons appelé «l'attitude de Québec», « à Londres d'abord, à Rome ensuite », Mgr Signay suggère de différer l'expédition des bulles jusqu'à ce qu'on ait obtenu l'assentiment de Londres.

${ }^{50}$ Il fallait alors deux mois pour qu'une dépêche parvint de Québec à Rome. La Propagande n'a donc pu recevoir celle de Mgr Signay avant la fin d'avril. 
Les ministres, ajoute-t-il, sont aujourd'hui mieux disposés qu'autrefois et une entente paraît possible ${ }^{51}$.

2. Le deuxième fait connu avec certitude est contenu dans la dépêche de Glenelg à Gosford du 26 mai: c'est la sympathie de Mgr Bramston, vicaire apostolique de Londres, pour le projet cher à Gosford et aux évêques du Canada.

Essayons maintenant de mettre un lien entre ces deux faits certains. Si, au reçu de la dépêche de Mgr Signay, Mgr Provencher avait été à Rome, il aurait été consulté; et, à notre avis, le départ des bulles n'aurait pas été retardé. Mais l'évêque de Juliopolis n'est plus à Rome; et la Propagande ne peut compter sur ses lumières. Laissé à lui-même et à ses conseillers ordinaires, le cardinal Fransoni retient la suggestion de $\mathrm{Mgr}$ Signay et décide d'entrer en pourparlers avec le ministre des colonies. Mgr Bramston aurait donc été mis au courant des décisions de la Propagande du 21 mars; il aurait été chargé d'explorer les dispositions du ministre et d'en informer le SaintSiège dans le plus court délai possible. L'intervalle qui sépare la dépêche de Glenelg à Gosford (26 mai) de l'expédition des bulles (18 juin) suffisait pour cela. Mais il va sans dire que le vicaire apostolique de Londres a pu être rassuré sur les dispositions favorables de Glenelg et rassurer Rome avant le 26 mai. D'ailleurs, la suggestion de Mgr Signay n'est pas de nature à surprendre le cardinal Fransoni. Un an plus tôt, le 28 avril 1835, il avait, de son propre chef, demandé à Mgr Bramston de s'enquérir des dispositions du ministre, relativement à l'érection d'un diocèse catholique à Montréal ${ }^{52}$. Le 2 juin, le vicaire apostolique avait répondu qu'il avait eu un entretien avec Glenelg; que celui-ci voudrait probablement connaître le sentiment du gouverneur général avant de se prononcer; que, du reste, une commission, chargée d'enquêter sur les problèmes du Canada, était sur le point de partir, qu'elle étudierait peut-être le problème de Montréal ${ }^{53}$. 1836.

${ }^{51} \mathrm{RAPQ}$ (1937-38), 85. Mgr Signay au cardinal Fransoni, 24 février

52 Prop. Lettere e decreti, 1835, vol 316, f. 300-301.

53 Prop. Scrit. rif. nei congressi. America Settentrional, Canada, 18311836 , vol. 3 , f. 37 . 
L'intervention de Mgr Bramston dans le débat, en 1836, nous paraît donc probable, parce qu'elle est conforme à la politique déjà connue du cardinal Fransoni. Enfin, il est digne de remarque qu'un contemporain, fort intéressé au problème, $\mathrm{Mgr}$ Provencher, admettait de la part de Mgr Bramston une intervention de dernière heure auprès de Glenelg. Il écrit, de Québec, le 2 août 1836 :

Il paraît que cette affaire de séparation a été traitée par la cour de Rome après mon départ, comme le suggérait la lettre de l'évêque de Québec. Peut-être que cette dépêche (celle de Glenelg du 26 mai) ou au moins la décision de la cour d'Angleterre aura été envoyée à Rome par l'évêque Bramston qui a traité cette affaire ${ }^{54}$.

Il nous semble, sauf meilleur avis, que dans sa dépêche du 26 mai à Gosford, Glenelg n'aurait pas fait état nommément de l'approbation de Mgr Bramston, s'il ne s'était pas agi d'une instance de celui-ci, récente et particulière, distincte de celle de 1835. N'aurait-il pas manqué à la prudence et à la discrétion diplomatiques en mentionnant le vicaire apostolique de Londres, sans savoir avec certitude s'il était encore aujourd'hui favorable à l'érection du diocèse de Montréal ? ${ }^{55}$

L'hypothèse que nous proposons ici a l'avantage de rendre compte de deux faits importants. Tout obstacle étant levé de la part du gouvernement impérial, on comprend mieux que Rome, 1836.

${ }^{54}$ AAM, Lettres de Mgr Provencher à Mgr Lartigue, Québec, 2 août

55 Pour transformer notre hypothèse en certitude, il nous manque deux documents, et deux documents essentiels: 1. la lettre de la Propagande demandant à Mgr Bramston d'intervenir auprès de Glenelg; 2. La lettre du vicaire apostolique de Londres informant la Propagande du succès de sa mission. Les recherches que nous avons faites ou fait faire par des archivistes tant au Canada qu'en Angleterre et à Rome n'ont pas donné de résultat positif. Seulement, l'expérience nous a appris, comme à tant d'autres chercheurs, que l'absence d'un document à un endroit où il devrait se trouver normalement n'est pas une preuve qu'il n'a jamais existé. Notre explication des faits nous paraît, à nous, certaine; mais nous la donnons pour ce qu'elle vaut. Elle respecte les connaissances acquises par ailleurs; elle est autorisée par la conduite qu'a tenue, un an plus tôt, le cardinal Fransoni, sur le même sujet; elle s'appuie enfin sur une tradition orale contemporaine, parvenue jusqu'à nous par Mgr Provencher, qui n'était pas un rêveur. 
après avoir retenu si longtemps les bulles, décide de les expédier. On comprend mieux aussi l'esprit qui anime la dépêche de Glenelg, l'intense désir qu'il manifeste de «satisfaire les catholiques du Bas-Canada sur ce sujet». Car il tient de $\mathrm{Mgr}$ Bramston que Rome a déjà érigé le diocèse de Montréal; et il tient de Gosford qu'il serait souverainement imprudent de s'opposer à cette décision. Quoi qu'il en soit, il nous paraît heureux que la reconnaissance civile ait été assurée avant l'arrivée des bulles. Autrement, il y aurait eu un choc désagréable entre Québec et Montréal. Mais dans l'ordre où les événements se sont produits, le conflit était impossible. Québec se flattait d'être arrivé au but sans avoir compromis le bon équilibre des relations entre l'Église et l'État; petit triomphe devant lequel Montréal fermait les yeux, car il avait l'essentiel, son indépendance, son autonomie. Pour comble de bonheur, Gosford est à Montréal au moment où les bulles y arrivent. Il a avec Mgr Lartigue des entretiens qui se déroulent dans une atmosphère de sympathie ${ }^{56}$. Il est au courant des événements qui se préparent: la prise de possession du siège de Montréal par Mgr Lartigue, le 8 septembre; et cela en vertu des bulles de Grégoire XVI ${ }^{57}$. Le 29 septembre, Mgr Lartigue était admis, en sa qualité d'évêque catholique de Montréal, à prêter le serment de fidélité au Roi ${ }^{58}$. Et le 2 décembre, Glenelg autorisait Gosford à le reconnaître pour les effets civils ${ }^{59}$.

L'histoire de l'érection du diocèse de Montréal n'est certes pas banale; elle est d'un intérêt palpitant, tant par les événements qui l'ont marquée que par les personnages qui y ont tenu un rôle. De plus, elle est souverainement importante par la libération qu'elle apportait à l'Église du Bas-Canada. Depuis la conquête, celle-ci avait été gênée, voire paralysée dans son développement par l'article du Traité de 1763, qui permettait

${ }^{56}$ Bull. Soc. Hist. de Saint-Boniface, III: 152. Mgr Provencher à Mgr Signay, Montréal, 26 août 1836. Nous savons par ailleurs que Gosford était encore à Montréal le 2 septembre.

57 Cette prise de possession, présidée par Mgr Provencher, eut lieu le 8 septembre.

58 RAPQ (1944-45), 209.

59 RAP (1931), 419. 
l'exercice de la religion catholique « en autant que le permettent les lois de l'Angleterre ». Cette restriction sera désormais inexistante. Londres ne cédera plus à la tentation d'intervenir dans la nomination des évêques ou dans la création des diocèses. Quelques fonctionnaires attardés, tel Ryland, continueront de faire appel à la loi de suprématie; ils ne seront entendus ni des gouverneurs généraux ni des ministres des colonies. Le gouvernement impérial se contentera d'approuver pour les effets civils les décisions de Rome et d'exiger des évêques catholiques le serment de fidélité au Roi. Quinze ans plus tard, cette dernière obligation aura elle-même disparu. Il est juste de dire la part de mérite qui revient à chacun dans cette histoire que nous venons de retracer. Saluons d'abord le clergé du district de Montréal et son vénérable chef, Mgr Jean-Jacques Lartigue. La ténacité avec laquelle ils ont défendu la cause leur confère un droit à notre admiration et à notre reconnaissance. L'attitude hésitante, et parfois déconcertante de Mgr Signay, ne doit pas nous empêcher de reconnaître la droiture de ses intentions. Il a su gagner, dès le début, et garder jusqu'à la fin la confiance de Gosford. Son ultime suggestion de différer l'expédition des bulles jusqu'après l'assentiment de Londres a produit d'heureux effets. Enfin, c'était une conjoncture heureuse que la présence de Gosford comme gouverneur général et celle de Glenelg au ministère des colonies. Ils ont contribué l'un et l'autre au succès final, à ce que nous avons appelé la libération de l'Église du Bas-Canada. Cette libération devait venir tôt ou tard. Mais il était à craindre qu'elle ne laissât de l'aigreur et de l'amertume dans les esprits, qu'elle ne retardât pour un temps le plein essor de l'Église. Grâce à Gosford et à Glenelg, le progrès s'est amorcé dans le calme et dans la paix.

\section{ULTIMES COMPLEMENTS}

Il restait à tirer les conséquences du nouvel état de choses et des excellentes dispositions de l'autorité civile. Ce sont les premiers gestes que pose Mgr Lartigue, en vertu de sa propre autorité, c'est-à-dire sans aucune dépendance de Québec. 


\section{LA SUCCESSION ÉPISCOPALE ASSUREE A MONTREAL PAR LA NOMINATION DE M. IGNACE BOURGET COMME COADJUTEUR}

Dès le 2 août 1836, soit avant même l'arrivée des bulles érigeant le diocèse, Mgr Provencher suggérait à Mgr Lartigue de se choisir un coadjuteur. C'était la dernière recommandation que lui avait faite Grégoire XVI. Le Pape avait même indiqué le mode à suivre dans le choix et la présentation du coadjuteur ${ }^{60}$. La suggestion est agréée. Le 12 novembre, Mgr Lartigue adressait à la Propagande les noms des trois candidats: M. Ignace Bourget, dignissimus, M. Hyacinthe Hudon, vicaire général et curé de Boucherville, dignior, et M. François-Xavier Demers, curé de Saint-Denis, dignus. Dans une lettre particulière au Souverain Pontife, Mgr Lartigue demandait nommément M. Ignace Bourget ${ }^{61}$. Il devait être exaucé. Datées du 18 mars 1837, les bulles arrivent à Montréal le 15 mai. Sans éclat et sans bruit, Mgr Lartigue et M. Bourget se rendent aussitôt à Québec où celui-ci est reçu au serment de fidélité à la Reine, en sa qualité de coadjuteur ${ }^{62}$. Un mandement du 20 mai apprenait l'heureuse nouvelle au diocèse et fixait au 25 juillet la cérémonie du sacre ${ }^{63}$. Le 27 juin, Glenelg communiquait l'approbation royale ${ }^{64}$. La reine, écrivait le secrétaire civil Walcott, a consenti à sanctionner la nomination de Mgr Bourget, «en autant qu'elle est compétente à le faire ou que la chose est nécessaire ${ }^{65} \gg$. La bonne et jeune Victoria - c'était la première année de son règne - n'a pas l'air de comprendre la nécessité des gestes qu'on lui demande de poser. Ou plutôt elle comprend qu'elle n'a rien à faire dans la nomination d'un évêque catholique. Que les temps sont changés !

Un double mérite revient ici à Mgr Lartigue; il a assuré la succession épiscopale à Montréal; et l'histoire proclame bien haut qu'en recommandant Mgr Bourget, il a très bien servi l'Église.

${ }^{60}$ AAM, Mgr Provencher à Mgr Lartigue.

61 RAPQ (1944-45), 218-219, 12 et 15 novembre 1836.

62 RAPQ (1944-45), 241.

63 Mand. Pastorales ... I : 10-12.

64 RAP (1931), 439.

65 AAM, Pièces et Actes, 3, f. 26. RAPQ (1944-45), 250. 


\section{L'INCORPORATION CIVILE}

Le 2 décembre 1836, Glenelg autorisait Gosford à reconnaître Mgr Lartigue comme évêque de Montréal. Une des conséquences de ce fait, c'était l'incorporation civile, qui ferait du diocèse une personne morale, capable d'acquérir et d'administrer les biens nécessaires à son existence. Cela, Glenelg le savait, il y consentait, désireux qu'il était de donner pleine et entière satisfaction aux catholiques. Mais le sujet était délicat. Aucun diocèse catholique n'avait encore été incorporé au Canada depuis la conquête; il importait que la chose se fît, et qu'elle se fît sans susciter l'animosité des protestants. C'est pourquoi le ministre indique sa pensée non dans la dépêche officielle, mais dans une lettre confidentielle à Gosford. Dans le cours ordinaire des choses, écrit-il, l'acte d'incorporation est du ressort de la Chambre d'assemblée; mais il pourrait arriver que celle-ci réservât ce projet de loi à la sanction spéciale de Sa Majesté; dans ce cas, le but poursuivi serait difficilement obtenu ${ }^{66}$. On comprend facilement la pensée du ministre: tenu d'encourager et de propager l'Église établie, c'est-à-dire l'anglicanisme, le gouvernement impérial pouvait-il approuver de gaieté de cœur une loi favorable à une autre religion, surtout au catholicisme ? Interprétant exactement la pensée de Glenelg, Gosford conseilla à Mgr Lartigue d'adresser sa demande directement à la Reine, non à la Législature ${ }^{67}$.

La Requête à Sa Majesté pour incorporation et amortissement de l'évêché de Montréal est du 1er mai 1837. Après un bref historique du Montréal ecclésiastique depuis 1821, elle note qu'en 1836, Mgr Lartigue a été reconnu par Londres comme évêque catholique du diocèse. Elle ajoute aussitôt

que, néanmoins, comme l'agrément de Votre Majesté pour un nouvel évêché suppose nécessairement une succession régulière d'évêques futurs pour remplir ce siège avec une dotation suffisante pour les faire

${ }^{66}$ RAP (1931), 419.

67 RAPQ (1944-45), 231. Mgr Lartigue à Gosford, 20 janvier 1837. Suivant le conseil de Gosford, l'évêque de Montréal adressera sa demande au Roi. Ce conseil, Gosford a dû le donner oralement. Du moins, nous n'en trouvons pas trace dans la documentation écrite. 
subsister et les rendre capables de s'acquitter des devoirs multiples de leur charge éminente; et vu que, dans la dépêche sus-mentionnée du 26 mai, Lord Glenelg a spécifié à Lord Gosford qu'il devait être bien entendu qu'en agréant cette nomination d'Evêques catholiques pour Montréal, le gouvernement ne pouvait attacher aucun émolument temporel quelconque, il est devenu indispensable, pour ne pas frustrer les intentions gracieuses de Votre Majesté en faveur de cet établissement, de pourvoir à son maintien et à sa prospérité par quelque autre moyen humain, mais légal, qui, sous la protection de la divine Providence, puisse le soutenir et le continuer ... ${ }^{68}$.

Mgr Lartigue demandait d'incorporer à la cathédrale SaintJacques les constructions et propriétés y attenant, pour être employées au bien du diocèse, pourvu que le revenu total ne dépasse pas la somme de deux mille livres sterling, cours d'Angleterre ${ }^{69}$. Gosford transmettait la requête à Londres, et le 30 décembre, Glenelg autorisait l'émission de Lettres patentes ${ }^{70}$. Mais cet ultime complément de l'érection du diocèse devait être, lui aussi, difficile, pour des raisons qui appartiennent à notre situation politique du moment et à la nonchalance des fonctionnaires. Quand l'autorisation de Glenelg arrive à Québec, Gosford est déjà parti pour l'Angleterre; il est remplacé temporairement par Colborne. Le 29 mai 1838, celui-ci cède la place à Durham. Après le départ de Durham, Colborne reprend les rênes de l'administration. Et pendant tout ce temps, Mgr Lartigue attend, mais en vain, une réponse à sa requête du 1er mai 1837. Il doit y voir là, pense-t-il, quelque «tricherie ${ }^{71}$ ». Et le 22 octobre, pour en avoir le cœur net, il charge son agent de Londres, Mgr Thomas Griffiths, vicaire apostolique, de s'informer auprès de Glenelg ${ }^{72}$. Et c'est de là que vint la lumière.

${ }^{68}$ AAM, Pièces et Actes, 3, f. 5-6 ${ }^{\mathrm{r}}$. RAPQ (1944-45), 239. Requête à Sa Majesté pour incorporation et amortissement de l'évêché de Montréal, 1er mai 1837.

69 Ibid.

70 RAP (1931), 471.

71 RAPQ (1945-46), 99. Mgr Lartigue à Mgr Signay, 8 mai 1839. 1838.

72 RAPQ (1945-46), 83. Mgr Lartigue à Mgr Griffiths, 22 octobre 
L'autorisation, datée de la fin de décembre 1837, avait été dûment envoyée à Québec; mais c'était au procureur général qu'il appartenait de rédiger les Lettres patentes ${ }^{73}$. Sachant désormais à quoi s'en tenir, Mgr Lartigue demande à Colborne de faire des recherches dans ses archives, afin de retrouver le document 《oublié » ${ }^{74}$. Les Lettres patentes sont datées du 15 août $1839^{75}$. Mais Mgr Lartigue ne peut vraiment chanter victoire que le 19 septembre ${ }^{76}$. Il n'est pas sans intérêt de constater que le témoin de cet acte d'incorporation a été le «vieux brûlot», sir John Colborne, lieutenant général et gouverneur des provinces du Canada.

\section{CONCLUSION}

A cause de la conjoncture politique - les Troubles de 1837 - qui a surtout retenu l'attention des historiens, on n'a peutêtre pas assez remarqué les progrès apportés à l'Église du Bas-Canada par l'érection du diocèse de Montréal. Par respect pour la loi de suprématie et pour ne pas paraître accorder au catholicisme une prééminence sur l'Église établie, Londres s'était toujours opposé à la création d'un deuxième diocèse catholique dans les limites du Bas-Canada. Montréal brisait l'obstacle et ouvrait une voie nouvelle en 1836. Désormais, l'autorité civile n'interviendra plus dans le gouvernement interne de l'Église.

De plus, l'érection du diocèse et sa reconnaissance par l'État venaient à temps. Il est heureux, en effet, que ces résultats aient été obtenus avant les Troubles de 1837. On sait que le recours aux armes fut noyé dans le sang, et que, pour empêcher le retour de pareils événements, les promoteurs de l'Union des Canadas à Londres et le gouverneur général, Poulett Thompson, avaient conçu le dessein d'assimiler les Canadiens français, c'est-à-dire d'en faire des Anglo-protestants. Dans la région de Montréal, théâtre de rébellion, la terrible répression avait

${ }^{73}$ AAM, Mgr Lartigue, Gouvernement anglais et évêché. Mgr Griffiths à Mgr Lartigue, Londres, 8 mars 1839.

74 RAPQ (1945-46), 98. Mgr Lartigue à Colborne, 30 avril 1839. août.

75 RAPQ (1945-46), 110. Incorporation de l'évêché de Montréal, 15 1839.

${ }^{76}$ RAPQ (1945-46), 114. Mgr Lartigue à Mgr Signay, 19 septembre 
accumulé les ruines, multiplié les deuils, semé, pour un temps, le désarroi, chez nos chefs politiques; et le peuple se demandait, non sans inquiétude, de quoi demain serait fait. Mais au milieu de la tempête, l'évêché et l'évêque de Montréal étaient restés debout comme un signe de ralliement pour des jours plus heureux. Il n'est pas possible de préciser la part qui lui revient dans la remontée qui s'imposait et qui ne manqua pas. Mais il est bien permis de se demander comment le secteur le plus populeux et le plus important du Canada français aurait pu, sans un chef spirituel à lui, triompher du danger de l'assimilation. Enfin, et ce point nous paraît d'une très grande importance, une fois l'orage passé, l'évêque de Montréal sera en place, respecté de l'autorité civile et jouissant auprès de l'immense majorité des fidèles d'un prestige agrandi: ce sera Mgr Bourget. Il refera l'unité, assurera au diocèse les institutions, les œuvres d'éducation et de charité, dont le besoin était impérieux et dont le rayonnement bienfaisant s'étend encore aujourd'hui à tout le Canada et même au-delà.

LÉON POULIOT, s.j., membre du Comité de direction de l'Institut d'Histoire de l'A.f.

Nota: Pour former des collections complètes de notre Revue, il nous manque les numéros suivants: I: nos 1-2-3; III: no 1 ; XI: no 1 ; XIII: nos $3-4$; XIV: nos 1-2. Vous rendriez grand service à la Revue en nous les offrant à un prix de rachat raisonnable.

La Direction 